\title{
EXCEPTIONAL SETS IN UNIFORM DISTRIBUTION
}

\author{
by R. C. BAKER \\ (Received 26th November 1977)
}

\section{Introduction}

Let $B$ be a measurable set of real numbers in $(0,1)$ of Lebesgue measure $|B|$ and let $x_{1}, \ldots, x_{n}$ be real. Then

$$
Z\left(B ; x_{1}, \ldots, x_{n}\right)
$$

denotes the number of $j(1 \leqq j \leqq n)$ for which the fractional part $\left\{x_{j}\right\} \in B$. The discrepancy of $x_{1}, \ldots, x_{n}$ is

$$
D\left(x_{1}, \ldots, x_{n}\right)=n^{-1} \sup _{I}\left|Z\left(I ; x_{1}, \ldots, x_{n}\right)-n\right| I||
$$

where the supremum is taken over all intervals $I$ in $[0,1]$.

Let $g_{1}(x), g_{2}(x), \ldots$ be a sequence of differentiable functions on the finite interval $[\alpha, \beta]$. Throughout the paper we assume that $g_{1}^{\prime}(x)$ and $g_{k}^{\prime}(x)-g_{j}^{\prime}(x)$ are positive and monotonic non-decreasing in $[\alpha, \beta]$ whenever $k>j \geqq 1$. We also assume that for some $p>0, C>0$,

$$
g_{k}^{\prime}(\beta) \leqq C k^{p} \quad(k \geqq 1),
$$

and that there are numbers $c>0$ and $a, 0 \leqq a<1$, such that

$$
g_{k}^{\prime}(x)-g_{j}^{\prime}(x) \geqq c
$$

whenever $j \geqq 1$ and $k \geqq j+C j^{a}$. Evidently $p \geqq 1-a$. We write

$$
F(B, n, x)=Z\left(B ; g_{\lambda}(x), \ldots, g_{n}(x)\right)-n|B|
$$

for $n \geqq 1, \alpha \leqq x \leqq \beta$, and

$$
D(m, n, x)=D\left(g_{m+1}(x), \ldots, g_{m+n}(x)\right)
$$

for $m \geqq 0, n \geqq 1$. In this paper we are interested in the exceptional sets

$$
E_{q}=\left\{x \in[\alpha, \beta]: \limsup _{n \rightarrow \infty} n^{q} D(0, n, x)>0\right\}
$$

and

$$
E(B)=\left\{x \in[\alpha, \beta]: \limsup _{n \rightarrow \infty} n^{-1}|F(B, n, x)|>0\right\}
$$

To make sure that $|E(B)|=0$ we consider only open sets $B$ with 'thin tail', that is

$$
B=I_{1} \cup I_{2} \cup \ldots \cup I_{n} \cup \ldots
$$


where $I_{1}, I_{2}, \ldots$ are the distinct component intervals of $B$ arranged in order of decreasing length, and

$$
b(B)=\liminf _{m \rightarrow \infty} \frac{\log \left|I_{m}\right|^{-1}}{\log m}>1 .
$$

The Hausdorff dimension of a real set $A$ is written $\operatorname{dim} A$.

Theorem 1. We have

$$
\operatorname{dim} E_{q} \leqq 1-(1-a-2 q) /(p+2 q) \quad\left(0<q<\frac{1}{2}(1-a)\right) .
$$

This improves my previous upper bound $(2,4)$, which was

$$
\min \left\{1-(1-a-2 q) /\left(p+2 q+\frac{1}{2}(1-a)\right), 1-(1-a-3 q) /(p+2 q)\right\} .
$$

Theorem 2. Let $f$ denote the polynomial

$$
f(y)=(b y-1)(p y+1-a-p)-p(3-y)(1-y) .
$$

For $b>1$ let $t=\max \left(b^{-1}, 1-(1-a) / p\right)$. Then, since $f(t)<0, f(1)>0$, and $f^{\prime}(y)>0$ $(t \leqq y \leqq 1)$, $f$ has a unique zero $\gamma$ in $(t, 1)$. We have

$$
\operatorname{dim} E(B) \leqq \gamma \quad \text { whenever } \quad b(B) \geqq b .
$$

Theorem 2 improves Theorem 5.1 of (3). Let $g_{j}(x)=a_{j} x$ where $a_{1}, a_{2}, \ldots$ is a strictly increasing sequence of positive integers. Then $a=0$ and

$$
f(y)=(b y-1)(p y+1-p)-p(3-y)(1-y) .
$$

In this particular case, it is shown in (3) that

$$
\operatorname{dim} E(B) \leqq \delta \quad \text { whenever } \quad b(B) \geqq b,
$$

where $\delta$ is the unique zero in $(t, 1)$ of the smaller function

$$
F(y)=(b y-1)(p y+1-p)-p(5-y)(1-y), \quad \text { so that } \delta>\gamma \text {. }
$$

It seems highly unlikely that Theorem 2 is best possible, but Theorem 1 might be. Some examples in Section 4 yield bounds beyond which the theorems cannot be improved.

Theorem 3. Let $\psi$ be a function on the positive integers such that

$$
K k^{-\gamma} \leqq \psi(k) \leqq 1 \quad(k=1,2 \ldots)
$$

for some $K>0$ and $\gamma, 0<\gamma<1$. Write $\Psi(n)=\sum_{k=1}^{n} \psi(k)$.

Let $a_{1}, a_{2}, \ldots$ be strictly increasing positive integers with

$$
a_{k} \leqq C k^{p} \quad(k \geqq 1)
$$

for some $C>0$ and $p \geqq 1$. Let $\alpha_{1}, \alpha_{2}, \ldots$ be real numbers. Write $N(n, x)$ for the number of solutions $k \leqq n$ of

$$
\left\{a_{k} x-\alpha_{k}\right\}<\psi(k)
$$


Then

$$
N(n, x) \sim \Psi(n) \quad \text { as } \quad n \rightarrow \infty
$$

except for a set of $x$ in $[0,1]$ of Hausdorf dimension at most

$$
1-(1-\gamma) /(p+2 \gamma) \text {. }
$$

Theorem 3 refines a result of LeVeque (Theorem 3 of (9)) that $N(n, x) \sim \Psi(n)$ for almost all $x$. I have little idea how far the upper bound obtained could be sharpened.

\section{Some lemmas}

In this section we collect together some preliminary results. Lemma 1 is similar to a result on p. 106 of (7). For a real set $A, A(\bmod 1)$ denotes the set of fractional parts $\{x\}(x \in A)$.

Lemma 1. Let $x_{1}, y_{1}, \ldots, x_{n}, y_{n}$ be real, then

$$
\left|D\left(x_{1}, \ldots, x_{n}\right)-D\left(y_{1}, \ldots, y_{n}\right)\right| \leqq 2 \max _{i}\left|x_{i}-y_{i}\right|
$$

Proof. Write $d=\max _{i}\left|x_{i}-y_{i}\right|$. Let $I$ be a subinterval of $[0,1]$ with endpoints $a, b$ $(a<b)$ and write $J=[a-d, b+d](\bmod 1)$. Then, if $K$ denotes the complement of $J$ in $[0,1]$,

$$
Z\left(J ; y_{1}, \ldots, y_{n}\right)+Z\left(K ; y_{1}, \ldots, y_{n}\right)=n=n(|J|+|K|)
$$

or

$$
Z\left(J ; y_{1}, \ldots, y_{n}\right)-n|J|=-\left(Z\left(K ; y_{1}, \ldots, y_{n}\right)-n|K|\right) .
$$

Either $J$ or $K$ is an interval, so

$$
Z\left(J ; y_{1}, \ldots, y_{n}\right)-n\left|J^{\prime}\right| \leqq n D\left(y_{1}, \ldots, y_{n}\right) .
$$

Now it is clear that

$$
\begin{aligned}
Z\left(I ; x_{1}, \ldots, x_{n}\right)-n|I| & \leqq Z\left(J ; y_{1}, \ldots, y_{n}\right)-n|I| \\
& \leqq Z\left(J ; y_{1}, \ldots, y_{n}\right)-n|J|+n(|J|-|I|) \\
& \leqq n D\left(y_{1}, \ldots, y_{n}\right)+2 n d .
\end{aligned}
$$

A similar argument shows that

$$
Z\left(I ; x_{1}, \ldots, x_{n}\right)-n|I| \geqq-n D\left(y_{1}, \ldots, y_{n}\right)-2 n d .
$$

Therefore

$$
n D\left(x_{1}, \ldots, x_{n}\right) \leqq n D\left(y_{1}, \ldots, y_{n}\right)+2 n d .
$$

Reversing the roles of $x$ 's and $y$ 's, the lemma follows.

Lemma 2. Let $F$ be a non-negative function on $[\alpha, \beta]$. Suppose

$$
|F(x)-F(y)| \leqq U|y-x| \quad(\alpha \leqq x \leqq \beta)
$$


and

$$
\int_{\alpha}^{\beta} F^{2}(x) d x \leqq V
$$

Let

$$
E=\{x \in[\alpha, \beta]: F(x) \geqq d>0\} .
$$

There is a covering of $E$ with intervals $J_{1}, \ldots, J_{h}$ such that for $0<\sigma \leqq 1$,

$$
\sum_{j=1}^{h}\left|J_{j}\right|^{\sigma}<C_{1}\left(1+U V d^{-3}\right)^{1-\sigma}\left(V d^{-2}\right)^{\sigma}
$$

where $C_{1}$ is a numerical constant.

Proof. This is a slight variant of Lemma 1 of (4).

Lemma 3. Let $g$ be a function on $[\alpha, \beta]$ whose derivative is monotonic nondecreasing with

$$
0<G \leqq g^{\prime}(x) \leqq H(\alpha \leqq x \leqq \beta) .
$$

Let $I$ be an interval in $[0,1]$ and let

$$
F=\{x \in[\alpha, \beta]:\{g(x)\} \in I\} .
$$

Then $F$ comprises intervals $J_{1}, \ldots, J_{m}$ with

$$
\sum_{j=1}^{m}\left|J_{j}\right|^{\sigma}<C_{2}|I|^{\sigma}\left(H^{1-\sigma}+G^{-\sigma}\right)
$$

for $0<\sigma \leqq 1$, where $C_{2}$ depends only on $\alpha, \beta$.

Proof. We have

$$
F=\left[u_{1}, v_{1}\right] \cup\left[u_{2}, v_{2}\right] \cup \ldots \cup\left[u_{m}, v_{m}\right]
$$

where $m \geqq 0, \alpha \leqq u_{1} \leqq v_{1}<u_{2}<v_{2}<\ldots<u_{m} \leqq v_{m} \leqq \beta$,

$$
\begin{gathered}
g\left(v_{j}\right)-g\left(u_{j}\right)=|I| \quad(1<j<m), \\
g\left(v_{j}\right)-g\left(v_{j-1}\right)=1 \quad(1<j<m), \\
\max \left(g\left(v_{1}\right)-g\left(u_{1}\right), g\left(v_{m}\right)-g\left(u_{m}\right)\right) \leqq|I| .
\end{gathered}
$$

Suppose for a moment that $m>2$. As $g$ is a convex function,

$$
\frac{g\left(v_{j}\right)-g\left(v_{j-1}\right)}{v_{j}-v_{j-1}} \leqq \frac{g\left(v_{j}\right)-g\left(u_{j}\right)}{v_{j}-u_{j}} \quad(1<j<m),
$$

or in view of (5), (6),

$$
v_{j}-u_{j} \leqq\left(v_{j}-v_{i-1}\right)|I| \quad(1<j<m) .
$$


Thus

$$
\begin{aligned}
\sum_{1<j<m}\left(v_{j}-u_{j}\right)^{\sigma} & \leqq|I|^{\sigma} \sum_{1<j<m}\left(v_{j}-v_{j-1}\right)^{\sigma} \\
& \leqq|I|^{\sigma}(m-2)^{1-\sigma}\left(\sum_{1<j<m}\left(v_{j}-v_{j-1}\right)\right)^{\sigma}
\end{aligned}
$$

using Hölder's inequality. But

$$
\begin{gathered}
\sum_{1<j<m}\left(v_{j}-v_{j-1}\right) \leqq \beta-\alpha, \\
m-2=g\left(v_{m-1}\right)-g\left(v_{1}\right) \leqq(\beta-\alpha) H
\end{gathered}
$$

in view of (4). Thus (even if $m \leqq 2$ )

$$
\sum_{1<j<m}\left(v_{i}-u_{i}\right)^{\sigma} \leqq|I|^{\sigma}(\beta-\alpha) H^{1-\sigma}
$$

Moreover,

$$
\left(v_{1}-u_{1}\right)^{\sigma}+\left(v_{m}-u_{m}\right)^{\sigma} \leqq 2\left(G^{-1}|I|\right)^{\sigma}
$$

from (4), (7). This proves the lemma.

Lemma 4. Let $B$ be an open set in $[0,1]$. Suppose there are measurable sets $G_{1}, G_{2}, \ldots$, such that

(i) $B$ is the union of $G_{m}$ and $m$ intervals $J_{m 1}, \ldots, J_{m m}$,

(ii) $c=\liminf _{m \rightarrow \infty} \frac{\log \left|G_{m}\right|^{-1}}{\log m}>0$.

Then $b(B) \geqq c+1$.

Proof. We have, for $\epsilon>0$,

$$
\left|G_{m}\right|<m^{-c+\varepsilon}
$$

for sufficiently large $m$. In the notation of (3), let $I_{j(1)}, \ldots, I_{j(m)}$ be the component intervals containing $J_{m 1}, \ldots, J_{m m}$ respectively. Then for large $m$,

$$
\begin{aligned}
m\left|I_{2 m}\right| \leqq \sum_{k>m}\left|I_{k}\right| & \leqq|B|-\left|I_{j(1)} \cup \ldots \cup I_{j(m)}\right| \\
& \leqq\left|G_{m}\right|<m^{-c+\epsilon}
\end{aligned}
$$

so that

$$
\left|I_{2 m}\right|^{-1}>m^{c+1-\epsilon}
$$

Obviously $b(B) \geqq c+1$.

Lemma 5. For $m \geqq 0, n \geqq 1$, we have

$$
\int_{\alpha}^{\beta}(n D(m, n, x))^{2} d x \leqq C_{3} n(m+n)^{a} \log ^{2}(n+1),
$$

where $C_{3}$ is independent of $m$ and $n$. 
Proof. This is established on p. 424 of (4).

We introduce the notation

$$
n_{k}=\left[\exp \left(k^{1 / 2}\right)\right] \quad(k \geqq 1) .
$$

The significant properties of this integer sequence are that $n_{k+1} / n_{k} \rightarrow 1$ as $k \rightarrow \infty$ and

$$
\sum_{k=1} n_{k}^{-\epsilon}<\infty
$$

for every $\epsilon>0$.

Lemma 6. We have

$$
D\left(0, n_{k}, x\right)<n_{k}^{-a}
$$

for sufficiently large $k$, except for a set of $x$ in $[\alpha, \beta]$ of Hausdorff dimension at most

$$
1-(1-a-2 q) /(p+q) \quad\left(0<q<\frac{1}{2}(1-a)\right) .
$$

Proof. It suffices to show that whenever

$$
1>\sigma>1-(1-a-2 q) /(p+q),
$$

the set $A(n)$ of $x$ in $[\alpha, \beta]$ for which

$$
D(0, n, x) \geqq n^{-q}
$$

can be covered by intervals $J(n, 1), J(n, 2), \ldots$ with

$$
\sum_{j=1}|J(n, j)|^{\sigma} \leqq C_{4} n^{-\epsilon} \quad(n \geqq 1)
$$

where $\epsilon>0$ and $C_{4}$ are independent of $n \geqq 1$. For then, given $K \geqq 1$, the set $A$ of $x$ belonging to infinitely many $A\left(n_{k}\right)$ can be covered by the family of intervals

$$
J\left(n_{k}, j\right)(j \geqq 1, k \geqq K) \text {. }
$$

We have

$$
\sum_{k \geq K} \sum_{j=1}\left|J\left(n_{k}, j\right)\right|^{\sigma} \leqq C_{4} \sum_{k \leq K} n_{k}^{-\epsilon} \rightarrow 0
$$

as $K \rightarrow \infty$, yielding $\operatorname{dim} A \leqq \sigma$, and indeed $\operatorname{dim} A \leqq 1-(1-a-2 q) /(p+q)$.

To get these coverings, we apply Lemma 2 with $F(x)=n D(0, n, x), d=n^{1-q}$, so that we may take

$$
V=C_{3} n^{1+a} \log ^{2}(n+1)
$$

in view of Lemma 5 , and

$$
U=2 n \max _{j \leq n} g_{j}^{\prime}(\beta) \leqq 2 C n^{p+1}
$$

in view of Lemma 1 . Thus $A(n)$ may be covered by intervals $J(n, 1), J(n, 2), \ldots$ with 


$$
\sum_{j=1}|J(n, j)|^{\sigma} \leqq C_{5} \log ^{2}(n+1)\left(n^{2+a+p-3(1-q)}\right)^{1-\sigma}\left(n^{1+a-2(1-q)}\right)^{\sigma}
$$

where $C_{5}$ is independent of $n$. We obtain (8) on noting that

$$
p+3 q-(1-a)<\sigma(p+q) \text {. }
$$

This proves Lemma 6.

Lemma 7. Let $h(m, n, x)$ ( $m \geqq 0, n \geqq 1)$ be functions satisfying the following conditions on $[\alpha, \beta]$ :

$$
\begin{gathered}
|h(m, n, x)-h(m, n, y)| \leqq d(m, n)|y-x|, \\
d(m, n) \sup |h(m, n, x)| \leqq C_{6} k^{\sigma} n^{\mu-\sigma} \quad(0 \leqq m \leqq k, 1 \leqq n \leqq k), \\
\int_{\alpha}^{\beta} h^{2}(m, n, x) d x \leqq C_{7} k^{\rho} n^{\nu-\rho} \quad(0 \leqq m \leqq k, 1 \leqq n \leqq k) .
\end{gathered}
$$

Here $C_{6}, C_{7}, \sigma, \mu, \nu, \rho$ are independent of $k, n$, with $\mu \geqq \sigma+1, \nu \geqq \rho+1$. Suppose further that

$$
n D(m, n, x) \leqq h(m, n, x) \quad(m \geqq 0, n \geqq 1, \alpha \leqq x \leqq \beta) .
$$

Then if $0<\lambda<\min \left(\frac{1}{2} \mu, \frac{1}{4}(\nu+\mu)\right)$ we have

$$
D(0, n, x)<n^{\lambda-1}
$$

for sufficiently large $n$, except for a set of $x$ of Hausdorf dimension at most

$$
(\mu+\nu-4 \lambda) /(\mu-2 \lambda)
$$

Proof. This is a slight variant of Theorem 4 of (4).

Lemma 8. Suppose that

$$
\limsup _{n \rightarrow \infty} n^{-1}|F(B, n, x)|>0
$$

Then

$$
\lim _{k \rightarrow \infty} \sup _{k} n_{k}^{-1}\left|F\left(B, n_{k}, x\right)\right|>0
$$

Proof. Let $n \geqq n_{1}$, then $n_{k} \leqq n \leqq n_{k+1}$ for some $k \geqq 1$. We clearly have

$$
F\left(B, n_{k}, x\right)+n_{k}|B| \leqq F(B, n, x)+n|B| \leqq F\left(B, n_{k+1}, x\right)+n_{k+1}|B|
$$

So that

$$
n_{k}^{-1} F\left(B, n_{k}, x\right)-\left(n_{k+1}-n_{k}\right) n_{k}^{-1} \leqq n^{-1} F(B, n, x) \leqq n_{k}^{-1} F\left(B, n_{k+1}, x\right)+\left(n_{k+1}-n_{k}\right) n_{k}^{-1} .
$$

If $n_{k}^{-1} F\left(B, n_{k}, x\right) \rightarrow 0$ as $k \rightarrow \infty$ then, in view of $n_{k+1} / n_{k} \rightarrow 1$ as $k \rightarrow \infty$, we evidently have $n^{-1} F(B, n, x) \rightarrow 0$ as $n \rightarrow \infty$. This proves the lemma. 
Lemma 9. Let $Q$ be a Borel set in $[\alpha, \beta]$ having Hausdorff dimension greater than $\sigma$, then there is a positive measure $\mu$ supported on $Q$ such that

$$
\mu([x, y]) \leqq(y-x)^{\sigma}(\alpha \leqq x<y \leqq \beta) .
$$

Proof. By Theorems 47 and 48 of (9), $Q$ has a compact subset of positive measure with respect to the function $t^{\sigma}$. The existence of $\mu$ now follows from Theorem 3 of Chapter II of (6).

\section{Proofs of Theorems 1 and 2}

The new idea in the proof of Theorem 1 is to use the smoothness of $D(m, n, x)$ (Lemma 1) rather than smoothness of a trigonometric sum that majorizes $D(0, n, x)$ (as in $(2,4)$ ).

Proof of Theorem 1. We apply Lemma 7, taking $h(m, n, x)=n D(m, n, x)$. In view of Lemma 5, we may take

$$
\rho=a, \quad \nu=1+a+\epsilon,
$$

for any $\epsilon>0$. In view of Lemma 1, we may take

$$
d(m, n)=2 n \max _{m+1 \leqq i \leqq m+n} g_{j}^{\prime}(\beta) \leqq 2 n(m+n)^{p},
$$

and thus (9) holds with

$$
\sigma=p, \mu=2+p .
$$

Write $\lambda=1-q$, where $0<q<\frac{1}{2}(1-a)$. The condition

$$
\lambda<\min \left(\frac{1}{2} \mu, \frac{1}{4}(\mu+\nu)\right)=\min \left(\frac{1}{2}(2+p), \frac{1}{4}(3+p+a+\epsilon)\right)
$$

is satisfied because $p \geqq 1-a$. Thus

$$
D(0, n, x)<n^{-q}
$$

for sufficiently large $n$, except for a set of Hausdorff dimension at most

$$
\frac{\mu+\nu-4 \lambda}{\mu-2 \lambda}=\frac{p+4 q-(1-a)+\epsilon}{p+2 q}
$$

Theorem 1 follows immediately.

In Theorem 2, the improvement of the result of (3) is obtained by the device of splitting $E(B)$ into two subsets, so that integrals

$$
\int_{\alpha}^{\beta} n^{2} D(m, n, x)^{2} d \mu(x)
$$

are no longer needed.

Proof of Theorem 2. Suppose that

$$
\eta=\operatorname{dim} E(B)>\gamma
$$


Then $f(\eta)>0$, and we can find a positive $d$ that satisfies

$$
\frac{p \eta+1-a-p}{3-\eta}>d>\frac{p(1-\eta)}{b \eta-1}
$$

We write, in the notation of (3),

$$
S(n)=\bigcup_{j \leqq n^{d}} I_{j}, T(n)=\bigcup_{j>n^{d}} I_{j}
$$

Thus

$$
F(B, n, x)=F(S(n), n, x)+F(T(n), n, x) .
$$

Now in view of Lemma 8 ,

$$
E(B)=\left\{x \in[\alpha, \beta]: \lim \sup _{k \rightarrow \infty} n_{k}^{-1}\left|F\left(B, n_{k}, x\right)\right|>0\right\} .
$$

It follows from (12) that

$$
E(B) \subset P \cup Q
$$

where

$$
P=\left\{x \in[\alpha, \beta]: \limsup _{k \rightarrow \infty} n_{k}^{-1}\left|F\left(S\left(n_{k}\right), n_{k}, x\right)\right|>0\right\}
$$

and

$$
Q=\left\{x \in[\alpha, \beta]: \limsup _{k \rightarrow \infty} n_{k}^{-1}\left|F\left(T\left(n_{k}\right), n_{k}, x\right)\right|>0\right\}
$$

We can readily estimate $\operatorname{dim} P$. We have

$$
F(S(n), n, x)=\sum_{j \leqq n^{d}}\left(Z\left(I_{j} ; g_{1}(x), \ldots, g_{n}(x)\right)-n\left|I_{j}\right|\right)
$$

so that

$$
n^{-1} F(S(n), n, x) \leqq n^{d} D(0, n, x) .
$$

It now follows from Lemma 6 that

$$
\operatorname{dim} P \leqq 1-(1-a-2 d) /(p+d) .
$$

But, from (11),

$$
\operatorname{dim} E(B)=\eta>1-(1-a-2 d) /(p+d) \geqq \operatorname{dim} P .
$$

Combining (13) and (14), it is clear that

$$
\operatorname{dim} Q \geqq \eta
$$

Now select a number $c, \eta^{-1}<c<b$, and a number $\sigma, c^{-1}<\sigma<\eta$, such that

$$
d>\frac{p(1-\sigma)}{c \sigma-1} \text {. }
$$

Since $Q$ is a Borel set having dimension greater than $\sigma$, there is a positive measure $\mu$ 
satisfying (10) supported on $Q$. We have

$$
\int_{\alpha}^{\beta} n^{-1}|F(T(n), n, x)| d \mu(x) \leqq \int_{\alpha}^{\beta} n^{-1} Z\left(T(n) ; g_{1}(x), \ldots, g_{n}(x)\right) d \mu(x)+\int_{\alpha}^{\beta}|T(n)| d \mu(x) .
$$

Now for large $n$,

$$
|T(n)|=\sum_{j>n^{d}}\left|I_{j}\right|<\sum_{j>n^{d}} j^{-c}<n^{-\epsilon}
$$

for some $\epsilon>0$. We need a similar estimate for

$$
\int_{\alpha}^{\beta} n^{-1} Z\left(T(n) ; g_{1}(x), \ldots, g_{n}(x)\right) d \mu(x) .
$$

Write $E(k, j)$ for the set of $x$ in $[\alpha, \beta]$ such that

$$
\left\{g_{k}(x)\right\} \in I_{j}
$$

Then

$$
\int_{\alpha}^{\beta} Z\left(T(n) ; g_{1}(x), \ldots, g_{n}(x)\right) d \mu(x)=\sum_{k=1}^{n} \sum_{j>n^{d}} \mu(E(k, j)) .
$$

We can estimate $\mu(E(k, j))$ by combining (10) with Lemma 3 and (1). We have

$$
\begin{aligned}
\mu(E(k, j)) & <C_{2}\left|I_{j}\right|^{\sigma}\left(C k^{p(1-\sigma)}+g_{1}^{\prime}(\alpha)^{-\sigma}\right) \\
& <j^{-c \sigma} n^{p(t-\sigma)} \quad\left(j>n^{d}, 1 \leqq k \leqq n\right)
\end{aligned}
$$

if $n$ is sufficiently large. Thus

$$
\begin{aligned}
\int_{\alpha}^{\beta} n^{-1} Z\left(T(n) ; g_{1}(x), \ldots, g_{n}(x)\right) d \mu(x) & <n^{p(1-\sigma)} \sum_{j>n^{d}} j^{-c \sigma} \\
& <2 n^{p(1-\sigma)-d(c \sigma-1)}
\end{aligned}
$$

The last exponent of $n$ is negative because of (16). Combining (17), (18) and (19), we certainly have

$$
\sum_{k=1}^{\infty} \int_{\alpha}^{\beta} n_{k}^{-1}\left|F\left(T\left(n_{k}\right), n_{k}, x\right)\right| d \mu(x)<\infty
$$

But then the series

$$
\sum_{k=1}^{\infty} n_{k}^{-1}\left|F\left(T\left(n_{k}\right), n_{k}, x\right)\right|
$$

converges for almost all $x$ with respect to $d \mu$. Since the series diverges at every point of $Q$, the support of $\mu$, we have a contradiction. This proves that

$$
\operatorname{dim} E(B) \leqq \gamma
$$

\section{Examples}

(i) Let $0<p \leqq 1, a=1-p$. By taking $g_{j}(x)=\left[j^{p}\right] x$, we show that the bound $4 q /(p+2 q)$ of Theorem 1 cannot be reduced below $2 q /(p+q)$. 


\section{EXCEPTIONAL SETS IN UNIFORM DISTRIBUTION}

If $x$ is real, write $w(x)$ for the supremum of all $\eta$ for which

$$
\liminf _{q \rightarrow \infty} q^{\eta}\|q x\|=0 .
$$

Here $\|\cdot\|$ denotes distance from the nearest integer. We write

$$
X(\eta)=\{x \in[0,1]: w(x) \geqq \eta\} .
$$

Then for $\eta>1$,

$$
\operatorname{dim} X(\eta)=2 /(\eta+1) .
$$

(This was proved by V. Jarnik and A. Besicovitch; (1) is the best reference).

Write $a_{j}=\left[j^{p}\right]$. Let $\epsilon>0$. The discrepancy of $a_{1} x, a_{2} x, \ldots, a_{n} x$ satisfies

$$
D(0, n, x)>n^{-\epsilon-p / \eta}
$$

for infinitely many $n$, whenever $x \in X(\eta)$. To see this, we follow the argument of Theorem 3.3 of (7), Chapter 2. Suppose $\epsilon<\eta / 2$. There are infinitely many positive integers $s$ and corresponding integers $t$ such that

$$
|x-t / s|<s^{-1-\eta+\epsilon}
$$

Write $n=\left[s^{(\eta-2 \epsilon) / p}\right]$. Then for $1 \leqq j \leqq n$,

$$
a_{j} x=k_{j} / s+\theta_{j}
$$

where $k_{j}$ is an integer and

$$
\left|\theta_{j}\right|<s^{-1-\epsilon} .
$$

The interval $I=\left(s^{-1-\epsilon}, s^{-1}-s^{-1-\epsilon}\right)$ thus contains none of the points $\left\{a_{1} x\right\}, \ldots,\left\{a_{n} x\right\}$, and therefore

$$
D(0, n, x) \geqq|I|>\frac{1}{2} s^{-1}>\frac{1}{4} n^{-p /(n-2 \epsilon)}
$$

for sufficiently large $s$.

It follows that

$$
X(\epsilon+p / q) \subset E_{q} \quad(0<q<p)
$$

for any $\epsilon>0$, and therefore

$$
\operatorname{dim} E_{q} \geqq 2 q /(p+q) \quad(0<q<p=1-a) .
$$

In case $p=1$, we can be more precise. The discrepancy of $x, 2 x, \ldots, n x$ satisfies

$$
D(0, n, x)<n^{e-1 / n}
$$

for sufficiently large $n$, unless $x \in X(\eta)$. This is Theorem 3.2 of (7), Chapter 2. In other words,

$$
E_{q} \subset X\left(q^{-1}-\epsilon\right)
$$

for $0<q<1,0<\epsilon<q^{-1}$. We easily deduce that

$$
\operatorname{dim} E_{q}=2 q /(1+q) \quad(0<q<1)
$$


(ii) Let $p=2, a=0$. By taking $g_{j}(x)=j^{2} x$, we show that the bound $(1+4 q) /(2+2 q)$ of Theorem 1 cannot be reduced below $2 q$.

If $x$ is irrational and $q_{1}, q_{2}, \ldots$ are the denominators of the continued fraction of $x$, write

$$
q_{k+1}=q \mu_{k}^{\mu_{k}} .
$$

Note that $\limsup _{k \rightarrow \infty} \mu_{k}=w(x)$ from the elementary theory of continued fractions. Let $k(1), k(2), \ldots$ be the indices for which $q_{k} \not \equiv 2(\bmod 4)$ and let

$$
\theta(x)=\limsup _{j \rightarrow \infty} \mu_{k(j)}
$$

The following result is easily deduced from Satz XIII of (5) using Koksma's inequality $(7, p .143)$. If $\theta(x)=\theta>1$, the discrepancy of $1^{2} x, 2^{2} x, \ldots, n^{2} x$ satisfies

$$
D(0, n, x)>n^{-\varepsilon-1 /(\theta+1)}
$$

for infinitely many $n$.

Now the techniques of (1) may easily be adapted to show that for $\eta>1$, the set $Y(\eta)=\{x \in[0,1]: \theta(x) \geqq \eta\}$ has dimension $2 /(\eta+1)$. Since (21) implies

$$
Y\left(q^{-1}-1+\epsilon\right) \subset E_{q}
$$

for $0<q<\frac{1}{2}, \epsilon>0$, we have

$$
\operatorname{dim} E_{q} \geqq 2 q \quad\left(0<q<\frac{1}{2}\right) .
$$

(iii) Let $b>1$. Let $a_{1}<a_{2}<\ldots$ be any integers with $a_{n+1} / a_{n} \rightarrow 1$ as $n \rightarrow \infty$, and let $g_{j}(x)=a_{j} x$. We shall show that there is an open set $G$ in $(0,1)$ with

$$
b(G) \geqq b, \quad \operatorname{dim} E(G) \geqq b^{-1} .
$$

With more calculation, our construction works for $a_{n}=\left[n^{p}\right](p>0)$. Thus the upper bound $\gamma$ of Theorem 2 could never be reduced below $b^{-1}$.

To construct $G$ we use the Cantor set $C(\rho)$, where $\rho$ is defined by

$$
\log 2 / \log \rho^{-1}=b^{-1},
$$

so that $0<\rho<\frac{1}{2}$.

If $J$ is the union of $m$ disjoint closed intervals $\left[\alpha_{i}, \beta_{i}\right]$, write $J^{p}$ for the union of

$$
\left[\alpha_{i}, \alpha_{i}+\left(\beta_{i}-\alpha_{i}\right) \rho\right],\left[\beta_{i}-\left(\beta_{i}-\alpha_{i}\right) \rho, \beta_{i}\right] \quad(1 \leqq i \leqq m) .
$$

Thus $J^{p}$ is the union of $2 m$ disjoint closed intervals.

Define $J(0), J(1), \ldots$ by induction as follows: $J(0)=[0,1], J(m)=J(m-1)^{\rho}(m>$ $0)$. We readily see that $J(m)$ is the union of $2^{m}$ disjoint closed intervals of length $\rho^{m}$. It is shown in (6), Chapter III that

$$
C(\rho)=\bigcap_{m=1}^{\infty} J(m)
$$

has Hausdorff dimension $\log 2 / \log \rho^{-1}$. We write $C^{\prime}(\rho)$ for the set of irrational numbers in $C(\rho)$ that are not endpoints of any interval of $J(m)(m \geqq 1)$. 
Our open set $G$ is

$$
G=\bigcup_{r=1}^{\infty} K_{r}
$$

where $K$, is the interior of the set $a_{r} J\left(a_{r}^{2}\right)(\bmod 1)$. The number of intervals comprising $K_{r}$ is at most $2^{a_{r}^{2}+1}$. Thus the set $\bigcup_{r=1}^{k} K_{r}$ comprises $h_{k}$ intervals, where

$$
h_{k} \leqq \sum_{r=1}^{k} 2^{a_{r}^{2}+1} \leqq k 2^{a_{k}^{2}+1} .
$$

Let $m$ be a positive integer, $m \geqq h_{1}$. Then for some $k=k(m)$,

$$
h_{k} \leqq m \leqq h_{k+1} \text {. }
$$

We can express $G$ as the union of $m$ intervals $J_{m 1}, \ldots, J_{m m}$ with the set

$$
G_{m}=\underset{r>k}{\cup} K_{r}
$$

Moreover, for large $m$,

in view of

$$
\begin{aligned}
\left|G_{m}\right| & \leqq \sum_{r>k}\left|K_{r}\right| \leqq \sum_{r>k} a_{r}\left|J\left(a_{r}^{2}\right)\right| \\
& \leqq \sum_{r>k} a_{r}(2 \rho)^{a_{r}^{2}}<a_{k}(2 \rho)^{a_{k}^{2}}
\end{aligned}
$$

$$
a_{j+1}(2 \rho)^{a_{1+1}^{2}}<\frac{1}{2} a_{j}(2 \rho)^{a_{j}^{2}} \quad \text { for large } j .
$$

Now

$$
\begin{aligned}
\frac{\log \left|G_{m}\right|^{-1}}{\log m} & \geqq \frac{-a_{k}^{2} \log 2 \rho-\log a_{k}}{\log h_{k+1}} \\
& \geqq \frac{a_{k}^{2}\left(\log \rho^{-1}-\log 2\right)-\log a_{k}}{\left(1+a_{k+1}^{2}\right) \log 2+\log (k+1)}
\end{aligned}
$$

so that

$$
\liminf _{m \rightarrow \infty} \frac{\log \left|G_{m}\right|^{-1}}{\log m} \geqq \frac{\log \rho^{-1}}{\log 2}-1=b-1 .
$$

It follows from Lemma 4 that $b(G) \geqq b$.

We now observe that if $x \in C^{\prime}(\rho)$, then $\{a, x\} \in K_{r}$ for $r \geqq 1$. Hence $\{a, x\} \in G$ for $r \geqq 1$. Obviously

$$
C^{\prime}(\rho) \subset E(G)
$$

and it follows that $\operatorname{dim} E(G) \geqq b^{-1}$.

\section{Proof of Theorem 3}

We use a lemma of a rather different nature from those in Section 2. Let $d(m)$ denote the number of divisors of a positive integer $m$ and $(s, t)$ the greatest common 
divisor of positive integers $s$ and $t$. If $I$ is an interval of the real line write $E_{I}$ for the union of all intervals $I+u$ ( $u$ integer) and $X(I, x)$ for the indicator function of $E_{l}$.

Lemma 10. For any intervals $J_{1}, \ldots, J_{n}$ of length $\leqq 1$,

$$
\int_{0}^{1}\left\{\sum_{k=1}^{n}\left(X\left(J_{k}, a_{k} x\right)-\left|J_{k}\right|\right)\right\}^{2} d x \leqq 2 \sum_{k=1}^{n}\left|J_{k}\right| d\left(a_{k}\right) .
$$

Proof. It is shown on p. 217 of (8) that

$$
\int_{0}^{1}\left\{\sum_{k=1}^{n}\left(X\left(J_{k}, a_{k} X\right)-\left|J_{k}\right|\right)\right\}^{2} d x \leqq 2 \sum_{k=1}^{n}\left|J_{k}\right| a_{k}^{-1} \sum_{j=1}^{k}\left(a_{j}, a_{k}\right),
$$

and on p. 219 of the same paper that

$$
\sum_{j=1}^{k}\left(a_{j}, a_{k}\right) \leqq a_{k} d\left(a_{k}\right)
$$

Lemma 10 follows on combining these two inequalities.

We introduce some further notations. Let $\rho(y, A)$ denote the distance from the real number $y$ to the set $A$. If $I$ is an interval with endpoints $a, b(a<b)$, and $\delta>0$, we write $I_{\delta}$ for the interval $[a-\delta|I|, b+\delta|I|]$. Define

$$
Y(I, \delta, x)=\max \left\{0,1-(\delta|I|)^{-1} \rho\left(x, E_{I}\right)\right\}
$$

and

$$
Z(I, \delta, x)=X\left(I_{\delta}, x\right)
$$

It is clear that for any real $x$,

$$
X(I, x) \leqq Y(I, \delta, x) \leqq Z(I, \delta, x) .
$$

Proof of Theorem 3. There are intervals $I_{1}, I_{2}, \ldots$, with $\left|I_{j}\right|=\psi(j)$ such that

$$
N(n, x)=\sum_{j=1}^{n} X\left(I_{j}, a_{j} x\right)
$$

Let $\epsilon>0$. We shall show that

$$
\underset{k \rightarrow \infty}{\lim \sup _{0}} \Psi\left(n_{k}\right)^{-1} \sum_{j=1}^{n_{k}} Y\left(I_{j}, n_{k}^{-\epsilon}, a_{j} x\right) \leqq 1
$$

except for a set $W$ of $x$ having dimension at most

$$
(p+3 \gamma+7 \epsilon-1) /(p+2 \gamma) \text {. }
$$

It follows from (22) and (23) that

$$
\limsup _{k \rightarrow \infty} \Psi\left(n_{k}\right)^{-1} N\left(n_{k}, x\right) \leqq 1
$$

outside $W$. Taking $\epsilon$ arbitrarily close to 1 we find that (24) holds outside a set of dimension at most $1-(1-\gamma) /(p+2 \gamma)$. A similar argument applies to $\liminf _{k \rightarrow \infty} \Psi\left(n_{k}\right)^{-1} N\left(n_{k}, x\right)$. We can now complete the proof by arguing as in Lemma 8 .

Thus it suffices to consider (23). 
Write

$$
M(n, x)=\max \left\{0, \sum_{j=1}^{n}\left(Y\left(I_{j}, n^{-\epsilon}, a_{j} x\right)-\int_{0}^{1} Z\left(I_{j}, n^{-\epsilon}, t\right) d t\right)\right\}
$$

and

$$
P(n, x)=\sum_{j=1}^{n}\left(Z\left(I_{j}, n^{-\epsilon}, a_{j} x\right)-\int_{0}^{1} Z\left(I_{j}, n^{-\epsilon}, t\right) d t\right) .
$$

Then in view of (22), whenever $M(n, x) \neq 0$ we have

$$
0<M(n, x) \leqq P(n, x),
$$

hence

$$
\int_{0}^{1} M(n, x)^{2} d x \leqq \int_{0}^{1} P(n, x)^{2} d x
$$

We now apply Lemma 10 , together with upper bounds for $d(m)$ and $a_{k}$, to get

$$
\int_{0}^{1} P(n, x)^{2} d x \leqq 2 \Psi(n)\left(1+2 n^{-\epsilon}\right) \max _{j \lesssim n} d\left(a_{j}\right)<\Psi(n) n^{\epsilon}
$$

for sufficiently large $n$. We also observe that for any $I, \delta>0$, and real $x, y$,

$$
Y(I, \delta, x)-Y(I, \delta, y)=\sum_{i=1}^{r} \int_{V_{i}} \pm(\delta|I|)^{-1} d t
$$

where $V_{1}, \ldots, V_{r}$ are intervals of total length $\leqq|y-x|$. Consequently if $x, y$ are real,

$$
\begin{aligned}
|M(n, x)-M(n, y)| & \leqq\left|\sum_{j=1}^{n}\left\{Y\left(I_{j}, n^{-\epsilon}, a_{j} x\right)-Y\left(I_{j}, n^{-\epsilon}, a_{j} y\right)\right\}\right| \\
& \leqq n^{\epsilon} \sum_{j=1}^{n}\left|I_{j}\right|^{-1} a_{j}|y-x| \\
& \leqq C K^{-1} n^{p+\gamma+1+\epsilon}|y-x|
\end{aligned}
$$

We now apply Lemma 2 with $[\alpha, \beta]=[0,1], F(x)=M(n, x), U=C K^{-1} n^{p+\gamma+1+\epsilon}, V=$ $\Psi(n) n^{\epsilon}$ and $d=\Psi(n) n^{-\epsilon}$. For large $n$ we have a covering of

$$
\left\{x \in[0,1]: \quad M(n, x) \geqq \Psi(n) n^{-\epsilon}\right\}
$$

by intervals $J_{n 1}, J_{n 2}, \ldots$ such that for $0<\sigma<1$,

$$
\sum_{i \leq 1}\left|J_{n j}\right|^{\sigma}<C_{8}\left(n^{p+\gamma+1+5 \epsilon} \Psi^{-2}(n)\right)^{1-\sigma}\left(n^{3 \epsilon} \Psi^{-1}(n)\right)^{\sigma}
$$

where $C_{8}$ is independent of $n$. Since

$$
\Psi(n)>n^{1-\gamma-\epsilon}
$$

for large $n$, we have

$$
\sum_{j \leq 1}\left|J_{n j}\right|^{\sigma}<C_{8} n^{p+3 \gamma-1+7 \epsilon-\sigma(p+2 \gamma)}
$$


If $\sigma>(p+3 \gamma+7 \epsilon-1) /(p+2 \gamma)$, the exponent of $n$ is negative. Arguing as in the proof of Lemma 6 it follows that

$$
M\left(n_{k}, x\right)<\Psi\left(n_{k}\right) n_{k}^{-\epsilon} \quad\left(k \geqq k_{0}(x)\right)
$$

except for a set of $x$ of dimension at most $(p+3 \gamma+7 \epsilon-1) /(p+2 \gamma)$. Since (25) implies (23), this completes the proof of Theorem 3 .

\section{REFERENCES}

(1) A. BAKER and W. M. SCHMIDT, Diophantine approximation and Hausdorff dimension, Proc. London Math. Soc. (3) 21 (1970), 1-11.

(2) R. C. BAKER, Slowly growing sequences and discrepancy modulo one, Acta Arith. 23 (1973), 279-293.

(3) R. C. BAKER, Khinchin's conjecture and Marstrand's theorem, Mathematika 21 (1974), 248-260.

(4) R. C. BAKER, Dyadic methods in the measure theory of numbers, Trans. Amer. Math. Soc. 221 (1976), 419-432.

(5) H. Behnke, Zur Theorie der diophantischen Approximationen, Abh. Math. Sem. Univ. Hamburg 3 (1924), 261-318.

(6) J-P. KAHANE and R. SALEM, Ensembles parfaits et series trigonometriques (Hermann, Paris, 1963).

(7) L. KUIPERS and H. NIEDERREITER, Uniform distribution of sequences (Wiley, New York, 1974).

(8) W. J. LEVEQUE, On the frequency of small fractional parts in certain real sequences III, J. für reine und angew. Math. 202 (1959), 215-220.

(9) C. A. Rogers, Hausdorf measures (Cambridge University Press, 1970).

Royal Holloway College

EGHAM

SURREY 